\title{
三视场星敏感器的多级星图识别算法
}

\author{
苟斌 ${ }^{1}$, 程咏梅 ${ }^{1}$ ，赵明艳 ${ }^{1}$ ，王会宾 ${ }^{1}$, 刘成 元 $^{2}$
}

(1.西北工业大学 自动化学院, 陕西 西安 $710129 ;$ 2.盲信号处理国家级重点实验室, 四川 成都 610041)

\begin{abstract}
摘 要: 为解决现有三视场星图识别算法效率慢、识别正确率低的问题,提出了一种面向三视场星敏 感器的多级星图识别算法:第一阶段利用单一可调参数的广义回归神经网络分别识别三幅单视场星 图; 第二阶段利用星库中存储的星间角距信息检验导航星识别结果,再以正确识别的导航星信息计算 星敏感器的 3 个视轴指向;第三阶段利用视轴指向辅助未识别与识别错误的导航星完成识别与校正; 最终, 以三视场内正确识别的导航星精确估计飞行器姿态信息。仿真结果表明, 当星点质心定位误差 的标准差达到 0.07 像素时, 该星图识别算法对实验样本的识别正确率仍高达 $98.9 \%$, 而识别时间仅为 $8.4645 \mathrm{~ms}$ 。同时, 由于提供求解姿态的星点信息较多且分布更广泛, 飞行器的三轴姿态估计精度也 随之提高。三视场星敏感器估计的飞行器偏航、俯仰、和滚转轴姿态精度分别为 $1.2058^{\prime \prime}, 1.0867^{\prime \prime}$ 以及 $1.2018^{\prime \prime}$ 。
\end{abstract}

关 键 词:三视场; 神经网络; 星图识别; 姿态 中图分类号:P128.4 文献标志码: A

天文导航系统利用导航星图像与导航星测量信 息估计载体相对天球坐标系的姿态 ${ }^{[1]}$ 。其中, 快 速、准确地识别导航星图是获取高精度姿态量测的 保障 ${ }^{[2-4]}$ 。由于恒星在天球坐标系的不均匀分布, 以及载体滚转轴姿态精度与导航星间的平均分离角 关系。单视场星敏感器的星图识别成功率与载体姿 态解算精度都不及多视场星敏感器 ${ }^{[5]}$ 。

传统多视场星敏感器的视场内星图识别方法需 要构造导航星空间匹配特征, 但多视场内的导航星 分布会导致匹配特征过于复杂与庞大 ${ }^{[6]}$; 视场间星 图识别方法则利用多视场的重叠区域融合多幅星图 为一副星图后再进行识别, 而多视场星敏感器多采 用小视场相机, 视场间的重叠区域有限。对此, 文献 [7] 提出了先视场内识别, 再视场间识别的多视场 星图识别策略。利用电子指南针估计星敏感器视轴 指向,构建局部识别数据库缩小视场间导航星匹配 范围。但电子指南针的低精度、三角形星图算法抗 星点质心定位误差效果差、视场间星图匹配复杂等 因素均对算法识别率和识别时间带来影响。
文章编号 : 1000-2758(2019)03-0541-06

为了提高三视场星敏感器的星图识别效率与识 别正确率, 本文以三个 $6^{\circ} \times 6^{\circ}$ 视场的正交三视场星 敏感器为研究对象, 改进先视场内识别, 再视场间识 别的星图识别策略, 提出三视场星敏感器的多级星 图识别算法。针对传统识别星图方法的缺陷, 将单 一可调参数的广义回归神经网络 ( generalized regression neural network, GRNN) 引人视场内导航星识 别 ${ }^{[8]}$; 利用星库中存储的星间角距检验上一步的识 别结果, 以正确识别的导航星估计 3 个视轴指向以 辅助未识别与识别错误的视场完成校正; 最终以正 确识别的导航星信息估计飞行器姿态。

\section{1 三视场星图多级识别算法}

\section{1 算法框架}

模拟的三视场星敏感器采用视场正交安装方 式,如图 1 所示。本文模拟了三视场星敏感器在天 球坐标系内的 1000 组随机姿态作为实验样本, 利 


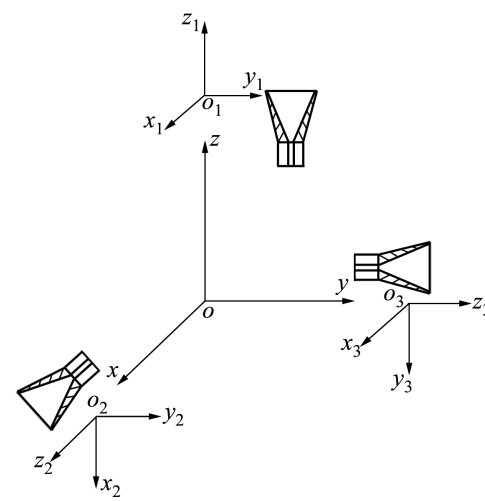

图 1 三视场星敏感器结构示意图

用 Smithsonian Astrophysical Observatory ( SAO $)^{[9]}$ 星 表中 6.5 星等以下的 6970 颗导航星信息模拟星图。 针对每组样本, 统计各视场从 $2^{\circ} \times 2^{\circ}$ 到 $12^{\circ} \times 12^{\circ}$ 变化 过程中可捕获的导航星数,结果如图 2 所示。

图 2 表明,在 1000 组 $6^{\circ} \times 6^{\circ}$ 三视场星图样本 中, 3 个视场捕获少于 3 颗星的概率分别为 $0.7 \%$, $1 \%$,及 $0.5 \%$, 但不存在 3 个视场同时捕获少于 3 颗 导航星的情况。为此, 本文提出多视场星敏感器多 级星图识别算法:第一阶段利用单一可调参数的 GRNN 算法进行视场内星图识别; 第二阶段以星库 中存储的星间角距信息检验导航星识别结果,仅当
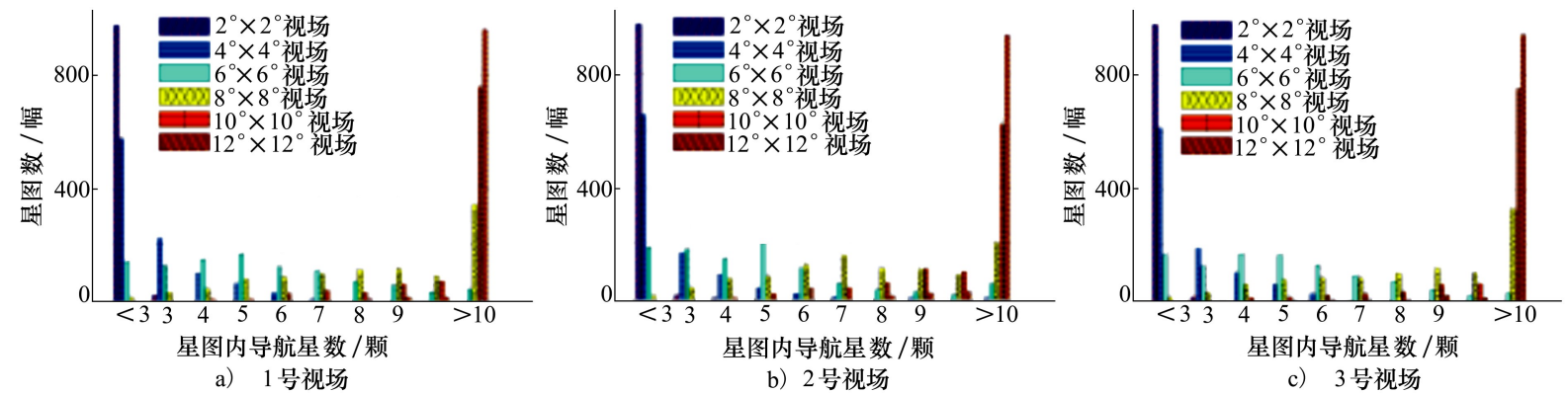

c) 3 号视场

图 2 视场变化下的导航星数统计

3 个视场均被正确识别时进行飞行器姿态估计, 否 则利用已正确识别的导航星估计星敏感器 3 个视轴 指向; 第三阶段利用视轴指向辅助未识别与识别错 误的导航星完成视场间识别与校正。

\section{2 视场内星图识别}

\section{1) 特征向量建立}

为了提高导航星识别正确率, 提升星图识别效 率, 本文构建了以 $\mathrm{P}$ 值向量为识别特征的神经网络 类星图识别算法 ${ }^{[10]}$ 。单一网络输人的 P 值向量可 以确保星图识别网络复杂度最小化 ${ }^{[11]}$ 。

假设最佳投影单位向量为 $\boldsymbol{\Omega}=\left[\begin{array}{lll}x & y & z\end{array}\right]^{\mathrm{T}}$ 。各导航 星的角距向量为 $\boldsymbol{d}_{i}=\left[d_{i_{-} 1} d_{i_{-2}} d_{i_{-} 3}\right]^{\mathrm{T}}, i=1,2, \cdots$, $N$ 。导航星总数 $N=6970$, 则每颗导航星的投影 点为

$$
P_{i}=x d_{i_{-} 1}+y d_{i_{-} 2}+z d_{i_{-} 3}=\boldsymbol{\Omega}^{\mathrm{T}} \boldsymbol{d}_{i}
$$

$P_{i}$ 的方差 $D(P)$ 越大, 投影轴上的投影点离散程度 最好。因此,最佳投影轴求解式为

$$
\begin{aligned}
& \max (D(P))=\max \left(\boldsymbol{\Omega}^{\mathrm{T}}\left(\frac{1}{N} \sum_{i=1}^{N} \boldsymbol{d}_{i} \boldsymbol{d}_{i}^{\mathrm{T}}\right) \boldsymbol{\Omega}-\bar{P}^{2}\right) \Leftrightarrow \\
& \max \left(\boldsymbol{\Omega}^{\mathrm{T}}\left(\frac{1}{N} \sum_{i=1}^{N} \boldsymbol{d}_{i} \boldsymbol{d}_{i}^{\mathrm{T}}\right) \boldsymbol{\Omega}\right)= \\
& \max \left(\boldsymbol{\Omega}^{\mathrm{T}} \boldsymbol{W} \boldsymbol{\Omega}\right)
\end{aligned}
$$

式中, $\bar{P}$ 为投影点 $P_{i}$ 均值; $W=\frac{1}{N} \sum_{i=1}^{N} \boldsymbol{d}_{i} \boldsymbol{d}_{i}^{\mathrm{T}}$ 。则

$$
\begin{gathered}
\max \left(\boldsymbol{\Omega}^{\mathrm{T}} \boldsymbol{W} \boldsymbol{\Omega}\right)=\max \left(\boldsymbol{\Omega}^{\mathrm{T}} \lambda \boldsymbol{\Omega}\right)= \\
\max \left(\lambda \boldsymbol{\Omega}^{\mathrm{T}} \boldsymbol{\Omega}\right)=\max (\lambda)
\end{gathered}
$$

最大特征根 $\lambda$ 对应的特征向量即为最佳投影轴。 通过对 6970 颗导航星的测试得到最佳投影轴为 [ $\left.\begin{array}{lll}0.397 & 0.580 & 0.711\end{array}\right]^{\mathrm{T}}$ 。每颗导航星根据公式(1) 可 求出对应的 $\mathrm{P}$ 值向量, 对该导航星进行编号, 并将 其 $\mathrm{P}$ 值与三边星角距信息一起存储在导航星库内。

2) GRNN 星图识别网络

引人单一可调参数的 GRNN 算法进行视场内 星图识别 ${ }^{[8]}$, 在保证星图识别正确率与识别效率的 同时解决传统神经网络星图识别算法过度依赖网络 参数的问题。GRNN 网络由输人层、隐层、输出层三 部分组成。隐层的基函数常采用高斯函数对输人信 号在局部产生响应。不同于传统神经网络, GRNN 的调节参数只有平滑因子 $\rho$ 一项。

为了确定最优 $\rho$ 的选取, 以上一节中 3000 幅 $6^{\circ} \times 6^{\circ}$ 三视场星图为实验样本。在各导航星质心定 位信息中加人 100 组均值为 0 ,标准差由 0 增至 0.1 像素的高斯误差; 每颗导航星以计算的 100 组 P 值 向量特征作为网络输人, 以 13 位二进制星号作为网 
络输出; 其次, $\rho$ 以 0.001 为步长, 由 0.001 增至 0.03 , 并用留 P 法 (leave-p-out, LPO) 对网络进行训练与测 试, 统计识别率 $e$; 最终, 确定最大识别率平均值对 应的 $\rho$ 为 GRNN 星图识别网络的平滑因子。

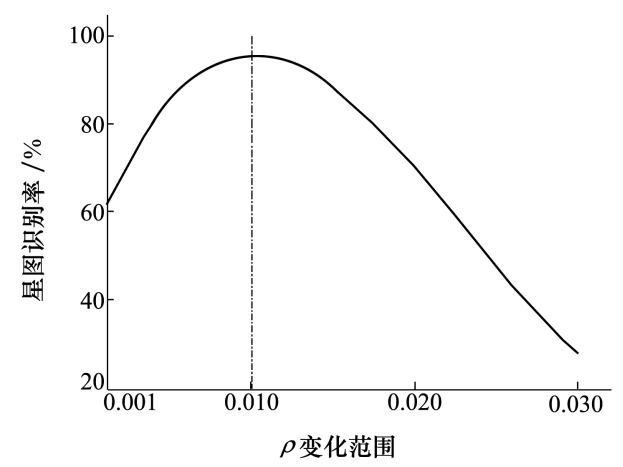

图 3 导航星识别率与 $\rho$ 的关系

图 3 表明, $\rho$ 过小会放大样本间的差异, 容易使 含有不同噪声的同一样本被误识别为不同样本; $\rho$ 过大会增加不同样本被误识别为同一种样本的概 率。根据统计结果, 本文选取最优平滑因子 $\rho=$ 0.01 。

\section{3 视场间星图识别与校正}

为了进一步提高识别正确率, 首先对视场内星 图识别结果进行检测。计算图中已识别导航星与其 最近星、次近星间三边星角距, 并与导航星库中记录 的标准三边星角距进行比对。角距门限 $\eta$ 满足 下式

$$
\eta=\sqrt{2} \delta \vartheta
$$

式中, $\delta$ 为星点的扩散半径, $\vartheta$ 为单像素空间张角。

再利用上一节中已正确识别的单视场星点信息 及星敏感器安装矩阵估计 3 个视场的视轴指向

$$
\left\{\begin{array}{l}
u_{1}^{i}=\left(\begin{array}{ll}
\boldsymbol{C}_{b}^{\mathrm{s} 1} & \boldsymbol{C}_{i}^{b}
\end{array}\right)^{\mathrm{T}}\left[\begin{array}{lll}
0 & 0 & 1
\end{array}\right]^{\mathrm{T}} \\
u_{2}^{i}=\left(\begin{array}{ll}
\boldsymbol{C}_{b}^{\mathrm{s} 2} & \boldsymbol{C}_{i}^{b}
\end{array}\right)^{\mathrm{T}}\left[\begin{array}{lll}
0 & 0 & 1
\end{array}\right]^{\mathrm{T}} \\
u_{3}^{i}=\left(\begin{array}{lll}
\boldsymbol{C}_{b}^{\mathrm{s}} & \boldsymbol{C}_{i}^{b}
\end{array}\right)^{\mathrm{T}}\left[\begin{array}{lll}
0 & 0 & 1
\end{array}\right]^{\mathrm{T}}
\end{array}\right.
$$

式中, $\boldsymbol{C}_{i}^{b}$ 是由已识别导航星估计的天球坐标系与载 体系间的转换矩阵; $u_{n}^{i}$ 为 $n$ 号视场的视轴指向 $(n=$ $1,2,3) ; \boldsymbol{C}_{b}^{\text {sn }}$ 分别为

$$
\begin{gathered}
\boldsymbol{C}_{b}^{s 1}=\left[\begin{array}{lll}
1 & 0 & 0 \\
0 & 1 & 0 \\
0 & 0 & 1
\end{array}\right], \boldsymbol{C}_{b}^{s 2}=\left[\begin{array}{ccc}
0 & 0 & 1 \\
0 & 1 & 0 \\
-1 & 0 & 0
\end{array}\right], \\
\boldsymbol{C}_{b}^{s 3}=\left[\begin{array}{ccc}
1 & 0 & 0 \\
0 & 0 & 1 \\
0 & -1 & 0
\end{array}\right]
\end{gathered}
$$

视轴指向误差范围最大值 $\xi$ 由星敏感器的姿态 估计误差 $\varphi$ e决定。根据欧拉位移定理 ${ }^{[12]}$, 当星敏 感器视轴方向垂直于误差欧拉轴时, 姿态估计误差 对视轴指向误差范围的贡献最大

$$
\cos \varphi_{e}=\frac{1}{2}\left[\operatorname{tr}\left(\boldsymbol{R}_{e}\right)-1\right]
$$

式中, $\operatorname{tr}\left(\boldsymbol{R}_{e}\right)$ 为姿态误差旋转矩阵 $\boldsymbol{R}_{e}$ 的迹。单视场 星敏感器解算的姿态中滚转轴的精度远低于其余两 轴, 本文主要考虑滚转误差旋转矩阵对星敏感器的 姿态估计误差的影响, 即

$$
\boldsymbol{R}_{e}=\left[\begin{array}{ccc}
1 & 0 & 0 \\
0 & \cos \theta & \sin \theta \\
0 & -\sin \theta & \cos \theta
\end{array}\right]
$$

根据 2.1 节中 1000 组 $6^{\circ} \times 6^{\circ}$ 三视场样本 $(3000$ 幅单视场星图) 解算的滚转姿态均方根误差的统计 结果。当星点质心添加了标准差为 0.07 像素的位 置误差。滚转姿态误差 $\theta$ 为 $0.998^{\circ}(1 \sigma)$, 最大视轴 指向误差范围为 $\xi=\varphi_{e}=3^{\circ}$ 。

二次候选导航星确定条件如下

$$
u_{\text {star }} u_{n}^{i} \geqslant \cos \left(R_{\mathrm{FOV}}+\xi\right) \quad n=1,2,3
$$

式中, $R_{\mathrm{FOV}}$ 为视场半径; $u_{\mathrm{star}}$ 为候选导航星方向矢 量,与导航星的天文信息赤经 (right ascension, Ra) 及赤纬 (declination, Dec) 有关

$$
\boldsymbol{u}_{\text {star }}=\left[\begin{array}{c}
\cos (R a) \cos (D e c) \\
\sin (R a) \cos (D e c) \\
\sin (D e c)
\end{array}\right]^{\mathrm{T}}
$$

根据公式 (9) 确定符合条件的候选导航星, 计 算各候选导航星间在天球坐标系下的空间星角距， 并与各未识别导航星与已识别导航星在载体系下星 角距逐一对比; 将满足星角距门限的候选导航星号 作为未识别导航星的识别结果。

\section{2 仿真与分析}

实验仿真环境为 Intel Core i5-6400 $2.7 \mathrm{GHz}$ PC 机; 三视场星敏感器仿真视场为 $6^{\circ} \times 6^{\circ}, \mathrm{CCD}$ 面阵像 素分辨率为 $512 \times 512$, 像素尺寸 $8 \mu \mathrm{m} \times 8 \mu \mathrm{m}$, 焦距为 $39.078 \mathrm{~mm}$; 星敏感器拍摄的星图是通过 Matlab2016a 软件根据 SAO 星表中星等小于 6.5 的 6970 颗恒星模拟生成。

\section{1 视场内星图识别结果分析}

本文模拟了 1000 组星敏感器在天球坐标系的 随机姿态作为实验样本。样本中每幅星图的星质心 
定位误差由 1 增至 0.07 个像素。图 4 统计了本文 算法、三角形匹配算法 (全天球识别) ${ }^{[11]}$ 、P 值向量 算法 ${ }^{[11]} 、 \mathrm{RBF}^{[2]}$ 以及 $\mathrm{BP}^{[3]}$ 星图识别算法在不同导 航星质心定位误差下的识别率以及平均识别时间。 其中, $\mathrm{RBF} 、 \mathrm{BP}$ 神经网络的训练集与 $\mathrm{GRNN}$ 相同。

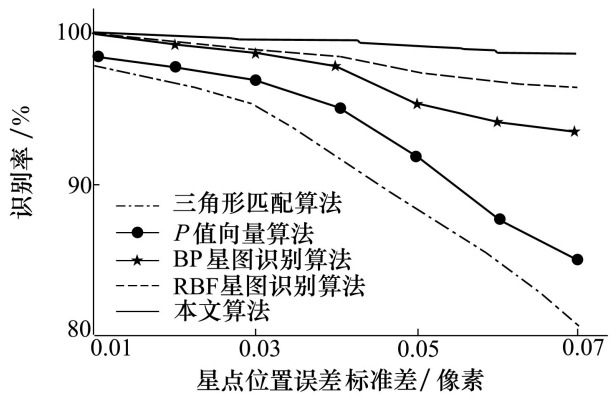

a) 不同星点位置误差下识别率对比

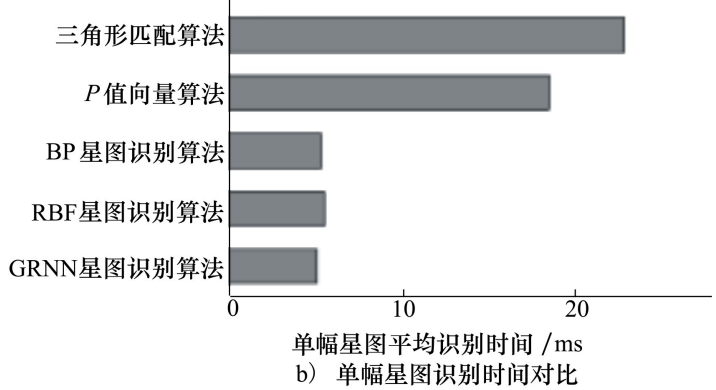

图 4 各识别方法性能对比

由图 4 可以看出,神经网络类方法具有相似的 星图识别时间, 且明显优于其他方法。但本文的 GRNN 星图识别算法在网络训练之初就考虑了星点 位置误差干扰, 增加了网络的容错能力。当星点位 置误差标准差为 0.07 像素时, 本文算法在 1000 组 实验样本中有 920 组 3 个视场被同时正确识别。

\section{2 视轴指向辅助视场间星图校正结果分析}

进一步分析上一节中含 0.07 像素星点质心定 位误差实验样本的识别结果。如图 5 所示, 1000 组 实验样本中至少有 1 个视场被正确识别的概率为 $100 \%$, 只有 2 个或 1 个视场被正确识别的样本组数 为 80 ,即 135 幅待识别星图。

每组样本利用已正确识别的导航星精确估计视 轴指向, 并建立局部导航星表进一步完成视场间星 图识别与校正。实验结果表明,除了 2 幅内无导航 星的星图外,本算法对 20 幅只包含有 1 或 2 颗导航 星的星图全部识别成功, 对 113 幅包含 3 颗及以上 导航星的误识别星图校正成功率达 $90.3 \%$, 该多级 星图识别算法使 1000 组三视场实验样本的整体识

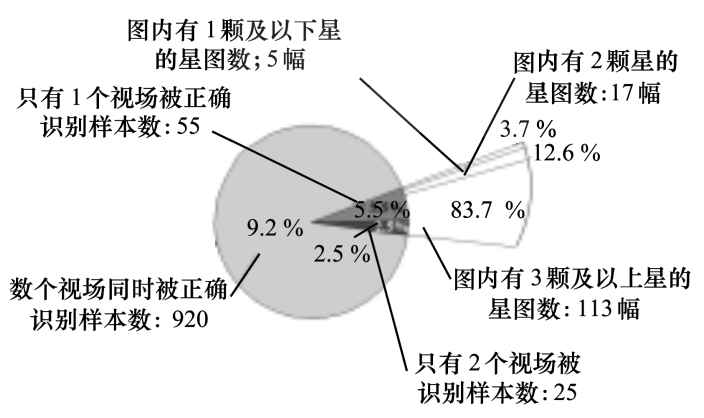

图 5 实验样品中各星图内导航星数统计

别正确率提升至 $98.9 \%$ 。

同时,本节还设计了 1 组与文献 [7]算法的对 比实验。实验采用 3.1 节中的 1000 次随机天球指 向作为实验样本, 文献 [7] 的视场内星图识别采用 三角形匹配算法,用于视场间星图识别的视轴指向 信息由仿真的电子指南针设备提供 (视轴服从均值 为 $0^{\circ}$, 标准差为 $2^{\circ}(1 \sigma)$ 的高斯误差 $)$ 。在相同导航 星质心定位误差条件下对比文献 [7]算法与本文算 法对 1000 组三视场实验样本的识别正确率和识别 时间。

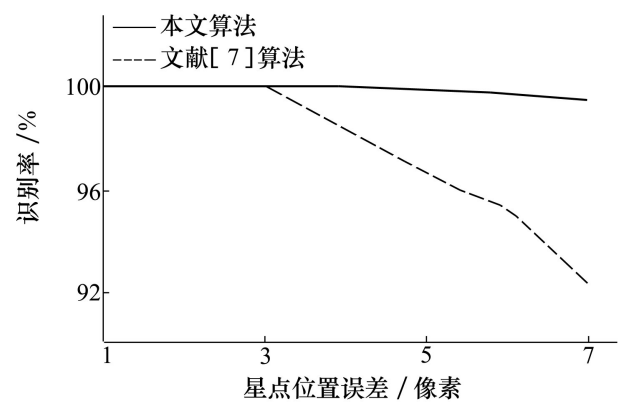

a）不同星点位置误差下识别率对比

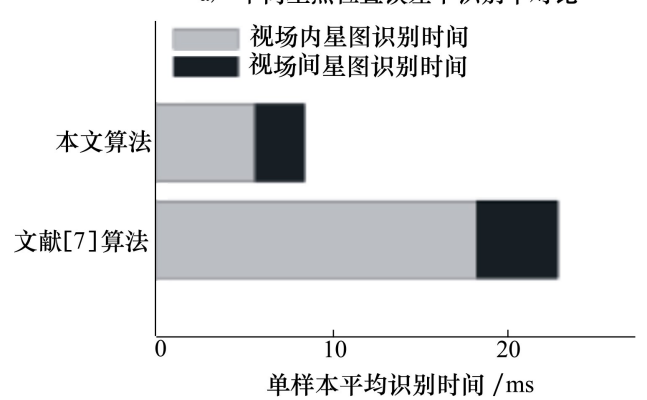

b) 单幅星图识别时间对比

图 62 种三视场星图识别算法性能对比结果

由图 6a) 可以看出, 随着导航星质心定位误差 的增大, 文献 [7]算法对实验样本的识别正确率也 
随之下降,这是由于三角形匹配算法对星角距识别 特征的变化较敏感。本文算法在训练星图识别网络 时就充分考虑了导航星质心定位误差的影响, 因此 对样本的识别结果优于文献 [7]算法。此外, 文献 [7]算法的平均识别时间为 $22.9563 \mathrm{~ms}$, 其中视场 内星图识别时间 $18.3652 \mathrm{~ms}$, 视场间星图识别时间 $4.5911 \mathrm{~ms}$ 。本文算法利用正确识别导航星估计的 视轴指向极大限制了视场间星图识别时导航星的搜 索范围。本文算法的平均识别时间为 $8.4645 \mathrm{~ms}$, 其中视场内星图识别时间 $5.6324 \mathrm{~ms}$; 识别结果检 测时间 $0.8231 \mathrm{~ms}$; 视场间星图识别与校正时 间 $2.0090 \mathrm{~ms}^{\circ}$

\section{3 姿态误差分析}

为了分析三视场星敏感器的定姿精度, 本实验 将该算法应用在轨高度 $500 \mathrm{~km}$; 轨道倾角 $45^{\circ}$; 地理 起始点为 $\left(0^{\circ} \mathrm{N}, 122.119^{\circ} \mathrm{E}\right)$ 的圆形卫星轨道。星敏 感器模拟器工作频率为 $5 \mathrm{~Hz}_{\text {。 }}$ 星图中星点质心提 取精度为 0.05 像素 ${ }^{[11]}$ 。本实验对比分析了单视场 星图与三视场星图的卫星姿态估计差异。

\section{表 1 姿态误差均方根误差}

\begin{tabular}{cccc}
\hline 视场 & 俯仰 $/\left({ }^{\prime \prime}\right)$ & 偏航 $/(")$ & 滚转 $/\left({ }^{\prime \prime}\right)$ \\
\hline 1 号视场 & 1.7035 & 2.8356 & 31.4555 \\
2 号视场 & 1.6982 & 2.8991 & 31.5069 \\
3 号视场 & 1.7063 & 2.9012 & 32.0335 \\
三视场 & 1.2058 & 1.0867 & 1.2018 \\
\hline
\end{tabular}

由表 1 可以看出, 3 个单视场解算的姿态精度 相似, 视场角的限制, 导致滚转轴方向的姿态估计精 度明显低于俯仰和偏航轴方向。三视场星敏感器提 供估计姿态的星点信息较多且空间分布更均匀, 三 轴姿态估计的精度也随之提高。偏航、俯仰和滚转 姿态估计精度分别为 $1.2058^{\prime \prime}, 1.0867^{\prime \prime}$ 以及 1.2018 ", 其中滚转轴姿态精度较 3 个单视场滚转轴 姿态精度分别提升了 $96.18 \%, 96.19 \%$ 及 $96.25 \%$ 。

\section{3 结 论}

本文提出了一种面向三视场星敏感器多级星图 识别算法,第一阶段利用神经网络进行视场内星图 识别, 第二阶段以星库中存储的星间角距信息检验 导航星识别结果, 当正确识别的视场数小于 3 时, 利 用已正确识别的导航星信息计算星敏感器的 3 个视 轴指向; 第三阶段利用视轴指向辅助未识别与识别 错误的导航星完成识别与校正。与文献 [7]相比, 本算法的视场内星图识别鲁棒性与识别时间具有明 显优势。当星点质心定位误差标准差为 0.07 像素 时, 本文算法对实验样本的识别正确率仍保持 $98.9 \%$, 识别时间仅为 $8.4645 \mathrm{~ms}_{\text {。 }}$ 三视场星图的高 识别率与合理的星点分布也使得飞行器偏航、俯仰、 滚转姿态精度分别提升为 $1.2058^{\prime \prime}, 1.0867^{\prime \prime}$ 以 及 $1.2018^{\prime \prime}$ 。

\section{参考文献:}

[1] 毛晓楠,梁为升,郑循江. 基于并行运算体系结构的星敏感器图像处理算法 [J]. 宇航学报, 2011, 32(3): 613-619 MAO Xiaonan, LIANG Weisheng, ZHENG Xunjiang. A Parallel Computing Architecture Based Image Processing Algorithm for Star Sensor[J]. Journal of Astronautics, 2011, 32(3) : 613-619 (in Chinese)

[2] 张少迪,王延杰,孙宏海. 三角剖分以及径向基函数神经网络在星图识别中的应用 [J]. 光学精密工程, 2012, 20(2): 395-402

ZHANG Shaodi, WANG Yanjie, SUN Honghai. Application of Triangulation and RBF Neural Network to Star Pattern Recognition [J]. Optics \& Precision Engineering, 2012, 20(2) : 395-402 (in Chinese)

[3] 马涛,孙红辉,肖松, 等. 基于 BP 神经网络的小样本星图识别方法 [J]. 应用光学, 2009, 30(2) : 252-256

MA Tao, SUN Honghui, XIAO Song, et al. Recognition Algorithm for Star Pattern of Little Swatch Based on BP Neural Network [J]. Journal of Applied Optics, 2009, 30(2) : 252-256 (in Chinese)

[4] 高社生,杨一, 高兵兵. 基于渐消滤波的神经网络优化算法及其应用 $[\mathrm{J}]$. 西北工业大学学报, 2015, 33(2) : 320-325 GAO Shesheng, YANG Yi, GAO Bingbing. Neural Network Learning Algorithm Based on Fading Kalman Filtering and Its Application [J]. Journal of Northwestern Polytechnical University, 2015, 33(2) : 320-325 (in Chinese)

[5] MIYATAKE K. Spacecraft Attitude and Body Rate Estimation with Four Star Sensor Heads: Concepts and Ground and On-Orbit Verification Results[C] // Automatic Control in Aerospace, 2010: 490-494

[6] 吴量,王建立,王吴京. 三视场天文导航视场间星图识别的导航星选取 [J]. 光学精密工程, 2015, 23(6): 1732-1741 
WU Liang, WANG Jianli, WANG Haojing. Guide Star Selection for Star Pattern Recognition between Three Fovs [J]. Optics and Precision Engineering, 2015, 23(6) : 1732-1741 (in Chinese)

[7] 王昊京,王建立, 吴量, 等. 基于粗测位置和方位的三视场快速星图识别方法 $[\mathrm{J}]$. 中国光学, 2014, 7(5)：768-778

WANG Haojing, WANG Jianli, WU Liang, et al. Fast Recognition on Star Pattern with Method of Three Fovs Based on Rough Location and Azimuth $[\mathrm{J}]$. Chinese Optics, 2014, 7(5) : 768-778 (in Chinese)

[8] 万士正, 常晓飞,间杰. 红外空空导弹气动光学传输效应分析方法 [J]. 西北工业大学学报, 2015, 33(4): 621-626

WAN Shizheng, CHANG Xiaofei, YAN Jie. Research Method of Aero-Optical Transmission Effect for IR Air to Air Missiles[J]. Journal of Northwestern Polytechnical University, 2015, 33(4) : 621-626 (in Chinese)

[9] WHIPPLE F L. Smithsonian Astrophysical Observatory Star Catalog[ J]. Philosophical Transactions of the Royal Society A Mathematical Physical \& Engineeringences, 1966, 284(1326) : 431-442

[10] 杨建,张广军,江洁. P 向量实现快速星图识别的方法 $[\mathrm{J}]$. 航空学报, 2007, 27(4): 756-762

YANG Jian, ZHANG Guangjun, JIANG Jie. Fast Star Identification Algorithm Using P Vector[ J]. Acta Aeronautica Et Astronautica Sinica, 2007, 28(4): 897-900 (in Chinese)

[11] 高晓光, 邸若海, 郭志高. 基于改进粒子群优化算法的贝叶斯网络结构学习 $[\mathrm{J}]$. 西北工业大学学报, 2014, 32( 5): 749-755

GAO Xiaoguang, DI Ruohai, GUO Zhigao. Bayesian Network Structure Learning Based on Improved Particle Swarm Optimization [J]. Journal of Northwestern Polytechnical University, 2014, 32(5) : 749-755 (in Chinese)

[12］张力军. 基于多视场星敏感器的航天器姿态确定方法研究 [D]. 长沙: 国防科学技术大学, 2011

ZHANG Lijun. Spacecraft Attitude Determination for Multiple Fields of View Star Sensors[D]. Changsha, National University of Defense Technology, 2011 (in Chinese)

\title{
Multi-Stage Star Image Identification Method of Three Field-of-View Star Sensor
}

\author{
GOU Bin ${ }^{1}$, CHENG Yongmei ${ }^{1}$, ZHAO Mingyan ${ }^{1}$, WANG Huibin ${ }^{1}$, LIU Chengyuan ${ }^{2}$ \\ $\left(\begin{array}{l}\text { 1.School of Automation, Northwestern Polytechnical University, Xi'an 710129, China; } \\ \text { 2. National Key Laboratory of Science and Technology on Blind Signal Processing, Chengdu 610041, China }\end{array}\right)$
}

Abstract: To improve the low efficiency and low navigation star identification rate of existing star image identification methods for three field-of-view (FOV) star sensor, a multi-stage star image identification method is proposed. Firstly, the generalized regression neural network which has only one adjustable parameter, is used to identify the star images in each field-of-view. Secondly, the star angular distance saved in the navigation star database is used to verify the identification results, and then the optical directions of the three FOVs are calculated by using the correctly identified navigation stars. Thirdly, the optical directions are utilized to auxiliary correct the unidentified and erroneous identified navigation stars. Finally, the high-accuracy probe attitude is estimated by using the correctly identified navigation stars in the three FOVs. The simulation results show that the identification rates of the experimental samples is of $98.9 \%$ when the standard deviation of star centroid positioning error increases to 0.07 pixels, but the identification time is only of $8.4645 \mathrm{~ms}$. Meanwhile, since the three field-of-view star sensor captures the more dispersed navigation stars, the probe attitude accuracy of yaw, pitch and roll angles by using the present method is improved evidently, which is of $1.2058^{\prime \prime}, 1.086$ 7", and $1.2018^{\prime \prime}$, respectively.

Keywords : three field-of-view; neural network; star image identification; attitude 